\title{
A construção de uma metodologia de atuação nos Arranjos Produtivos Locais (APLs) no estado do Ceará: um enfoque na formação e fortalecimento do capital social e da governança
}

\author{
The Construction of a Methodology for Action in the Local Productive \\ Arrangements (LPAs) in the State of Ceará: Highlighting the Training and \\ Strengthening of Social Capital and of Governance
}

La construcción de una metodología de actuación en los Arreglos Productivos Locales (APLs) en el estado de Ceará: un enfoque en la formación y gortalecimiento del capital social y de la gobernanza

\author{
Mônica Alves Amorim* \\ Maria Vilma Coelho Moreira** \\ Ana Silvia Rocha Ipiranga*
}

Recebido em 28/11/03; revisado e aprovado em 15/08/04; aceito em 23/08/04.

\begin{abstract}
Resumo: Os Arranjos Produtivos Locais (APLs) constituem uma forma incipiente de organização mais sistêmicas das pequenas empresas. O desenvolvimento do APL liga-se a sua transformação em uma estrutura mais complexa como o Sistema Produtivo Local (SPLs), onde capital social e governança são determinantes para a expansão da interdependência dos atores envolvidos. Esse trabalho visa estruturar uma tecnologia de mobilização dos atores, de modo a organizá-los em rede e desencadear um processo de mudança.

Palavras-chaves: Arranjos Produtivos Locais; capital social; governança.
\end{abstract}

Abstract: The Local Productive Arrangements (LPAs) constitute an incipient form of organisation more systemic than small companies. The development of the LPA is connected to its transformation into a more complex structure such as the Local Productive System (LPSs), where social capital and governance are determinants for the expansion of the interdependence of the involved actors. This study seeks to structure a technology for the mobilisation of actors that they can be organized into a network and unchain a process of change.

Key words: Local Productive Arrangements; social capital; governance.

Resumen: Los Arreglos Productivos Locales (APLs) constituyen una forma incipiente de organización más sistémicas de las pequeñas empresas. El desarrollo del APL se relaciona a su transformación en una estructura más compleja como el Sistema Productivo Local (SPLs), donde capital social y gobernanza son determinantes para la expansión de la interdependencia de los actores aliados. Ese trabajo visa estructurar una tecnología de movilización de los actores, de modo a organizarlos en red y desencadenar un proceso de cambio.

Palabras clave: Arreglos Productivos Locales; capital social; gobernanza.

\section{Introdução}

$\mathrm{O}$ fortalecimento de micro e pequenas empresas (MPEs) coloca-se como uma sólida alternativa para o alcance do almejado desenvolvimento com inclusão social. Sabese, entretanto, que as MPEs enfrentam sérias limitações para concorrer com empresas de maior porte, tendo esse problema se agravado ainda mais com o processo de globalização, que acirrou a concorrência ao aproximar os competidores instalados em outras fronteiras aos mais remotos mercados. Ao mesmo tempo, é bem menos difundida a idéia de que a essência das dificuldades que cerceiam as MPEs relaciona-se não ao tamanho dessas, mas sobretudo à forma como costumeiramente funcionam, ou seja isoladas. Diferentemente das grandes empresas, as MPEs não podem se permitir à comodidade do isolamento. Isto porque, operando escalas de produção reduzidas, as MPEs não conseguem auferir economias de escala, ficando assim presas a condições ineficientes de produção. Além disso, o isolamento das MPEs acentua suas limitações para o desenvolvimento de capacidade inovativa, cada vez mais essencial para a obtenção de vantagens competitivas.

Dentre as abordagens que se propõem para analisar as MPEs, encontram-se aquelas denominadas de Distritos Industriais, Clusters e Sistemas e Arranjos Produtivos Locais. Este estudo priorizará a última abor-

\footnotetext{
* Professora do Departamento de Teoria Econômica da Universidade Federal do Ceará (monicaaa@secrel.com.br). * Professoras do Mestrado em Adminstração de Empresas da Universidade de Fortaleza (vmoreira@unifor.br; anasilviaipi@uol.com.br).
} 
dagem citada, apresentando uma estratégia de mobilização dos atores integrantes dos Arranjos Produtivos Locais (APLs), de modo a possibilitar a participação e atuação conjunta (capital social) e a coordenação e controle das ações e projetos encaminhados (governança). Todo o esforço parte de uma abordagem participativa com o fim de estruturar e implementar uma tecnologia social voltada para a mudança e o desenvolvimento dos APLs. Nesse trabalho de mobilização dos atores dos APLs, deve-se incluir, além dos agentes produtivos, a participação de representantes do poder local e de outros níveis com relevância para a especialidade do APL, instituições com atuação nos territórios dos APLs, organizações associativas e comunitárias relacionadas ao negócio em foco. Além da mobilização dos atores, a estratégia deve abordar o encaminhamento dos projetos priorizados pelos atores mobilizados, de forma a garantir a sistematicidade das ações e o estabelecimento da governança dos APLs. A partir deste objetivo, foi formulada a questão norteadora deste trabalho: por meio de quais instrumentos a tecnologia de mobilização dos APLs, visando à construção e/ou fortalecimento da governança, poderá ser operacionalizada?

Outrossim, a importância desse trabalho reside na ampla disseminação do conceito de arranjo produtivo entre os diversos órgãos governamentais (federal, estadual e municipal) do país, os quais têm anunciado prioridade para essas estruturas produtivas, todavia sem ainda contar com uma forma objetiva de atuação. Na verdade, mesmo com diferentes programas e recursos alocados para os APLs, já mapeados em todo o Brasil, não se percebe ainda uma estratégia clara e eficiente para abordar os APLs, voltada para a formação do capital social e governança dos mesmos. Ao contrário, parece que tudo ocorre ainda como na forma tradicional quando se enfocavam, de forma estanque, setores ou grupos de empresas. Tratando-se de uma estrutura em que o desenvolvimento do todo é mais importante do que o desenvolvimento das partes e, portanto, onde o entrosamento dos atores e sinergia de ações apresentam-se como essenciais, os APLs devem ser abordados através de um modelo específico que contemple esses as- pectos. É justamente essa lacuna que este estudo pretende preencher. Inicialmente será apresentada relevante literatura que trata das diversas correntes acerca de aglomerações de empresas. Em seguida, a tecnologia de mobilização dos APLs será apresentada, seguida das considerações finais e sugestões para futuras pesquisas.

\section{Reflexões teóricas}

O processo de globalização da economia tem ocasionado um redesenho das forças produtivas. A expansão de novas formas flexíveis de organização da produção aponta para a falência do modelo fordista, trazendo conseqüências importantes no âmbito econômico, social, organizacional e tecnológico.

A chamada "especialização flexível" destaca-se assim como um novo modelo de organização industrial, com exemplos bem sucedidos na Terceira Itália, Alemanha, França, que tiveram como base a expansão de pequenas e médias empresas, cuja adaptabilidade às flutuações de demanda, ao dinamismo inovador tornaram-nas organizações importantes neste novo modelo industrial que se tem delineado nas últimas décadas (BAPTISTA, 2002) ${ }^{1}$.

O conceito de "especialização flexível" nos reporta a outro conceito de aglomerações industriais elaborado por Alfred Marshall (1996), o "distrito industrial", que engloba aspectos, tais como: firmas especializadas, territorização, mercado de trabalho e cooperação. $O$ ressurgimento da idéia do "distrito marshalliano" ocorre no recente período de reestruturação produtiva, decorrente do esgotamento dos modelos taylorista/fordista, que ocasionaram, além do aumento do desemprego formal, a precarização do trabalho e o crescimento das economias informais. Em contrapartida, o tipo de distrito industrial marshalliano, acena para a possibilidade de construção de um locus de cooperação, baseado na confiança e em aspectos sócio-culturais, formado pelas redes de interação entre os agentes.

Apesar do forte otimismo que causou frente à desestruturação de formas clássicas de organização da produção, o modelo de distrito industrial também tem apresentado sinais de esgotamento. Como ressaltam 
Schmitz e Nadvi (1999) e Le Borgne (1991), o distrito industrial italiano, exemplo na literatura do modelo marshalliano, começa, sobretudo nos anos 90, a apresentar mudanças na sua dinâmica de funcionamento. A cooperação cede lugar para formas de contratualização entre firmas, a constituição de firmas líderes, a especialização em produtos de baixa qualidade e o emprego de mão de obra pouco qualificada (AMARAL FILHO, 1999).

De fato, há diversas abordagens que analisam aglomerações de empresas. Dentre elas, destacam-se: o modelo formalizado por Krugman (1998), a abordagem da economia de empresas, na qual destaca-se $M$. Porter (1998), as discussões sobre os clusters, com A. Scott (1988), da economia de inovação, com a contribuição destacada de D. B. Audrestch (1988), e a abordagem de pequenas empresas, distritos industriais, com destaque de H. Schmitz (1999).

Todas estas vertentes analíticas utilizam intrinsecamente conceitos da abordagem de Sistema Produtivo Local, que já tem sido usado, de forma mais estruturada em países desenvolvidos, e de maneira mais incipiente em países em desenvolvimento, no que se refere a estratégias de desenvolvimento regional e local.

Os estudos de Economia Regional enfatizam prioritariamente aspectos relativos aos "fatores locacionais" que influenciam a implantação de uma indústria em determinada área geográfica, e seus desdobramentos na reprodução e transformação de regiões geo-econômicas específicas.

Já a abordagem da estratégia de cluster segue uma tendência porteriana (M. Porter) e situa-se na fronteira entre a literatura de organização industrial e desenvolvimento regional e demonstra que a análise setorial não consegue captar a complexidade dos fenômenos que envolvem a dinâmica industrial. No entanto, a análise de clusters procura captar os elementos estruturais e sistêmicos da aglomeração enfatizando a rivalidade entre as firmas e quais os fatores da sua dinâmica interna que afetam a competitividade dos agentes. Assim, a análise de clusters de empresas prioriza o estudo da sua estrutura investigando-se, por exemplo: o tamanho de seus membros, as articulações entre eles, os padrões de especialização e concorrência e as vantagens competitivas que podem ser criadas a partir da estruturação dessa modalidade de aglomeração.

Estas abordagens apresentam alguns pontos confluentes e complementares, pois enfatizam a proximidade geográfica dos agentes produtivos, e a relevância do contexto social e institucional como fatores importantes na consolidação dessas aglomerações (BRITTO e ALBUQUERQUE, 2002). Contudo, a abordagem de cluster se aproxima mais da grande produção flexível do que da pequena, diferenciando-se tanto do modo fordista de produção, baseado na grande indústria de produção de massa, como também da visão do distrito industrial marshalliano, da pequena produção flexível. Além de enfatizar mais a concorrência do que a cooperação entre os membros do cluster, esta abordagem também atribui relevância à formação de uma indústria-chave ou indústriaschave numa determinada região, transformando-as em líderes de mercado. As empresas chamadas âncoras estimulam sem dúvida, o desenvolvimento da região, através da mobilização de agentes produtivos localizados no mesmo território, porém o crescimento mais homogêneo dos agentes produtores passa a sr secundário.

Diante da diversidade de vertentes teóricas acerca do desempenho competitivo das empresas, tem-se chegado a uma convergência das percepções de que o foco de análise não deve ser centrado apenas na empresa individual, mas, sobretudo nas relações entre as firmas e entre estas e as instituições que interagem com elas num determinado espaço geográfico. Ademais, a necessidade de buscar eficiência e competitividade exige que as MPEs se organizem em torno de uma forma própria de organização, distinta daquele praticada pela grande empresa. $\mathrm{Na}$ impossibilidade de auferirem economias de escala dentro das próprias empresas, quando se ocupam, ao mesmo tempo, das várias etapas do processo de produção, as MPEs podem, alternativamente, obter economias de escala se especializando em uma ou apenas algumas etapas do processo produtivo. A especialização, complementada pela cooperação praticada entre diversos agentes concentrados em um certo território, constitui a base dos chamados arranjos produti- 
vos locais (APLs)². Entretanto, a formação dos APLs mesmo constituindo um avanço comparado ao funcionamento disperso e isolado de empresas e outros agentes, não deve ser um objetivo definitivo, mas apenas uma transição para uma forma superior de organização, mais sistêmica, sustentável e com maior nível de interdependência entre os agentes, ou seja, os sistemas produtivos locais (SPLs) $)^{3}$. Este novo foco tem possibilitado a reorientação de formas de intervenção do poder público na promoção da política industrial e tecnológica. De acordo com Cassiolato e Szapiro (2002, p. 11):

A abordagem de arranjos e sistemas produtivos locais tem a proposta de elaborar um modelo que englobe categorias tradicionais nas análises de aglomerações como a cooperação, mas que inclui os processos de aprendizado, capacitação e inovação, considerados crescentemente como fundamentais para a sustentação da competitividade dos agentes participantes de aglomerações de empresas.

Ao lado das empresas, são também protagonistas dessas formas organizacionais outros atores locais, como governos, associações e instituições de financiamento, ensino, formação, pesquisa e outras atividades correlatas. Nessas formas de organização (APL e SPL), a especialização, além de aumentar a escala de produção de cada empresa, favorece a produção compartilhada, o que, por sua vez, estimula a cooperação e a inovação. Essas relações sócio-econômicas passam a fazer parte do processo de produção, e assim, dão origem à formação de um tecido sócio-produtivo, onde os agentes se especializam, cooperam, trocam informações, aprendem e compartilham de um projeto comum: o desenvolvimento do conjunto das empresas. A passagem do enfoque da empresa individual para o dos APLs desloca o centro da análise para as relações entre as empresas e, entre estas e diversas instituições que atuam em um determinado espaço geográfico (território). A unidade de análise deixa então de ser a empresa isolada e passa a ser a comunidade de empresas - o APL. Daí a importância de se estimular as ligações entre os diversos atores do APL.

A transformação dos APLs em SPLs envolve um salto de complexidade relacio- nado à ampliação da interdependência entre os diversos agentes (econômicos, políticos, institucionais e sociais) que pode ser alcançada através das práticas de cooperação, cultura participativa e adoção sistemática de ações coletivas. A expansão dessas práticas exige o fortalecimento da confiança dos agentes que atuam no território, de modo a permitir que os agentes se prontifiquem a encaminhar em conjunto projetos de interesse comum. A capacidade de unir esforços para trabalhar em conjunto (capital social) assume grande importância em estruturas como APLs e SPLs, pois o desenvolvimento desses depende de ações coletivas, em oposição a ações individuais dos agentes. Assim, o esforço de evolução dos APLs para SPLs não pode prescindir da construção e fortalecimento do capital social.

O fortalecimento do capital social está condicionado a criação de uma rede de cooperação entre atores e instituições. Na medida em que as ações deixam de ser cada vez menos individualizadas e tornam-se mais coletivizadas, torna-se necessária a constituição de uma força de coordenação das diversas ações de modo a garantir a formação de sinergias que possam garantir o alcance dos objetivos desejados. A intensificação das relações entre essas partes e o estabelecimento da coordenação dessas relações promove a boa governança, atributo necessário à evolução do estágio de APL para SPL. Por sua vez, o surgimento da boa governança depende de um aprendizado que pode ser derivado da repetição e consistência das ações coletivas. Portanto, estes dois aspectos, o fortalecimento do capital social e a formação da boa governança, surgem como ingredientes essenciais para a consolidação e transição do estágio de APL para SPL. Importa assim explorar formas de intervenção que possibilitem a construção e consolidação desses ativos.

Este quadro de referência constitui-se importante ferramenta para a compreensão da dinâmica e funcionamento dos arranjos e sistemas produtivos locais. Vale ressaltar que a definição de arranjo enfatiza os aspectos ineficientes em relação à inovação, assim como as interações entre os próprios agentes do arranjo, tornando-se importante uma investigação sobre tais interações a fim de se possibilitar a compreensão mais apro- 
fundada dos níveis de inovação e aprendizagem existentes nos arranjos produtivos possibilitando uma forma de se entender melhor sua trajetória e evolução.

\section{Tecnologia de mobilização dos APLs}

Partindo-se de uma visão sistêmica, os APLs devem ser analisados considerando as dimensões produtivas, institucionais e comu- nitárias do território. Destacam-se assim três vertentes que contribuem para o incremento da competitividade e os avanços na sustentabilidade de um APL: o desenvolvimento da capacidade produtiva, a formação e fortalecimento do capital social e boa governança, e a formação de competências e o aprendizado dos seus agentes, como ilustrado a seguir:

\section{Diagrama 1: As vertentes do processo de desenvolvimento do APL}
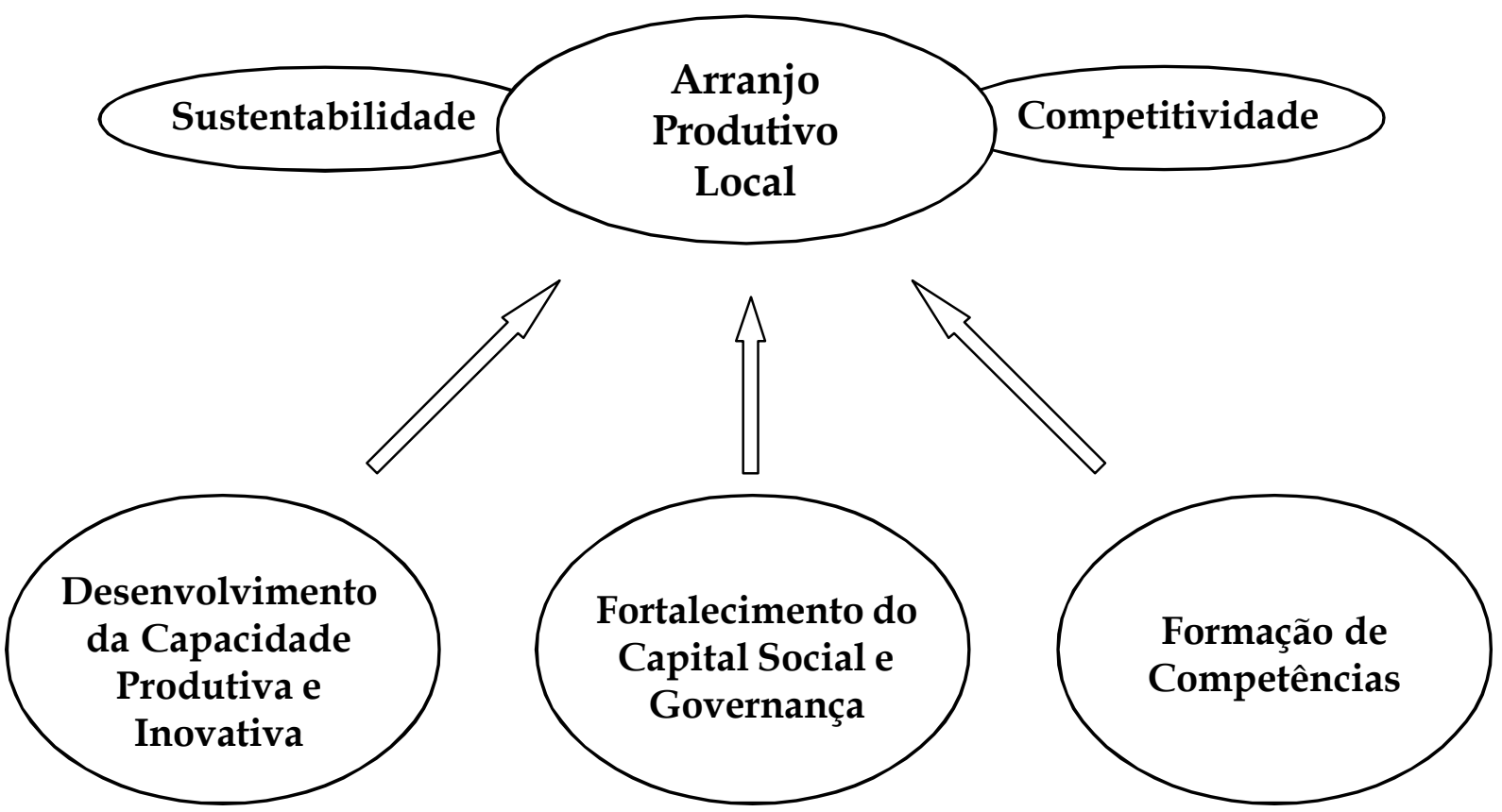

\subsection{Capacidade produtiva e inovativa}

O desenvolvimento da capacidade produtiva e inovativa envolve melhorias na qualidade dos produtos e processos, o adensamento das aglomerações e o aprofundamento da especialização, bem como a inovação e a diferenciação dos produtos. Esses passos mostram-se estratégicos para um forte posicionamento do APL no mercado, caracterizado por uma concorrência cada vez mais acirrada.

\subsection{Capital social}

O capital social é de grande importância quando se considera a atividade produtiva inserida em um território composto de um aglomerado de agentes que precisam unir sinergias para trabalhar em conjunto. O fortalecimento do capital social está condicionado a criação de uma rede de cooperação entre atores e instituições. A intensificação das relações entre essas partes e a formação da coordenação dessas relações promove a boa governança.

\subsection{Formação de competências}

A formação de competências também se apresenta como fundamental na conquista da competitividade e sustentabilidade, dado que o processo de evolução de Arranjo Produtivo para Sistema Produtivo envolve o alcance de novos níveis de complexidade. Assim a competência que se busca corresponde a inteligência prática de situações que se apóiam sobre os conhecimentos ad- 
quiridos e os transformam com quanto mais força quanto mais aumentam as complexidades das situações. Tal competência implica em saber como mobilizar, integrar e transferir os conhecimentos, recursos e habilidades em um contexto determinado. Assim, as habilidades dos agentes produtivos devem também ser trabalhadas para que os mesmos se capacitem para responder satisfatoriamente aos desafios impostos pelo mercado.

\subsection{Abordagem cooperativa}

A passagem de um estágio de arranjo produtivo para uma estrutura mais sistêmica requer a formação da governança, entendida como um mecanismo de controle e coordenação do desenvolvimento do território. A construção da governança pode ser facilitada pela criação de entidades organizacionais que contribuam para uma ambiência comunitária favorável ao desenvolvimento dos APLs. Os objetivos dessas entidades estão direcionados para a exploração das potencialidades do capital social através da valorização e criação de sinergias entre as competências locais, a organização das complementaridades entre recursos e projetos, as trocas de saberes e de experiências, a formação de redes de ajuda mútua, a autoorganização das comunidades locais, a maior participação dos atores e da população em geral nas decisões políticas, a abertura para novas formas de especialidades e parcerias como meios para viabilizar o desenvolvimento local do território.

O modelo de tecnologia de mobilização dos APLs sugere, assim, a criação de três tipos de entidades que juntas contribuirão para o fortalecimento do capital social e da governança nos territórios dos APLs. Referidas entidades constituem instrumentos de mobilização social e se baseiam em uma abordagem cooperativa. Parte-se da identificação de um grupo maior de atores selecionados ("Fórum para a Mudança") que, a partir do seu funcionamento, deve ser desdobrado em diversos grupos de trabalho ("Laboratórios para Inovação") com foco em tarefas específicas apontadas como prioritárias para a resolução dos problemas do APL, devendo esses possuir mecanismos explícitos de ligação com instituições de referência ("Pontos de Escuta"), portadoras do estado da arte referente a temas de preocupação específica. Abaixo estão descritas as características dessas entidades.

\subsubsection{Fórum para a mudança}

Trata-se de um espaço organizacional e inovador através do qual os atores institucionais públicos e privados serão convidados a participar de um programa de mudanças. É um lugar de encontro e de difusão de idéias com o fim de assegurar uma relação entre as propostas de inovação e aqueles que serão chamados à gestão e a atuação das práticas específicas a cada contexto.

\section{Quadro 1: Composição do fórum para mudança}

\begin{tabular}{|l|}
\hline \multicolumn{1}{|c|}{ Exemplos de Composição do Fórum para a Mudança } \\
\hline Atores Selecionados \\
\hline 1) Lideranças do APL \\
\hline 2) Representante da Prefeitura \\
\hline 3) Representante do SEBRAE-Serviço Brasileiro de Apoio às Micro e Pequenas \\
Empresas \\
\hline 4) Representante do Banco do Nordeste do Brasil-BNB \\
\hline 5) Representante do Governo Estadual \\
\hline 6) Membro do Clube dos Diretores Logistas-CDL local \\
\hline 7) Representante do Banco do Brasil \\
\hline 8) Representante das Universidades com atuação no território \\
\hline 9) Representante dos Centros Tecnológicos do Ceará - CENTEC/Centro de \\
Vocação Tecnológica - CVTs presentes no território \\
\hline 10) Transportadoras, Correios \\
\hline 11) Representante dos compradores e intermediários dos produtos do APL. \\
\hline Fonte: Elaborado pelas autoras
\end{tabular}




\subsubsection{Laboratórios para a inovação}

Trata-se de grupos de trabalho operativos ativados para colocar em prática as propostas de melhorias discutidas e decididas nos Fóruns. Os laboratórios são formados por representantes selecionados do fórum e deverão oferecer soluções para assuntos específicos aos participantes do fórum. Cada grupo operará em um arco de tempo definido. Os laboratórios terão natureza e modalidades diferentes dependendo dos objetivos.

\section{Quadro 2: Objetivos dos laboratórios para inovação}

\begin{tabular}{|l|}
\hline \multicolumn{1}{|c|}{ Exemplos de Laboratórios e Seus Objetivos } \\
\hline 1. Observatório nacional e internacional sobre as novas tendências, tecnologias,etc \\
\hline $\begin{array}{l}\text { 2. Criação de um pool para a prospeção de novos mercados, canais de distribuição } \\
\text { nacionais e internacionais }\end{array}$ \\
\hline 3. Desenvolvimento de práticas cooperativas (relações e trocas) interempresas e interarranjos \\
\hline $\begin{array}{l}\text { 4. Relações com o crédito bancário e desenvolvimento de formas de financiamento } \\
\text { customizados e coletivo }\end{array}$ \\
\hline 5. Ações focalizadas no processo de inovação, design e diversificação \\
\hline 6. Projetos comuns para a formação e desenvolvimento dos Recursos Humanos \\
\hline $\begin{array}{l}\text { 7. Desenvolvimento de novas competências técnicas (skills). Exemplos: design, logística e } \\
\text { marketing }\end{array}$ \\
\hline 8. Gestão de problemas comuns do ambiente \\
\hline $\begin{array}{l}\text { 9. Criação de uma rede de parcerias com autoridades públicas e governo estadual, municipal } \\
\text { e federal }\end{array}$ \\
\hline $\begin{array}{l}\text { 10. Coesão e interação entre vários sujeitos produtivos e institucionais (nacionais e } \\
\text { internacionais) para trocas de best-practices }\end{array}$ \\
\hline $\begin{array}{l}\text { 11. Ações de marketing social e cultural relacionadas com o desenvolvimento da imagem } \\
\text { (aspectos intangíveis) do território }\end{array}$ \\
\hline 12. Promoção de ações comerciais entre as PME e entre os Arranjos \\
\hline 13. Estímulo ao empreendedorismo e o controle de PME emergentes \\
\hline 14. Relações entre contratadas e sub-contratadas \\
Fonte: Elaborado pelas autoras
\end{tabular}

\subsubsection{Pontos de escuta}

O objetivo é de criar uma rede de interlocutores da comunidade local e regional, envolvendo as pessoas e as organizações na avaliação e operacionalização dos projetos que experimentarão práticas e procedimentos inovadores. A partir dos Pontos de Escuta, os Laboratórios se familiarizam com o estado da arte pertinente aos temas por esses tratados.

\section{Quadro 3: Pontos de escuta}

\begin{tabular}{|l|}
\hline \multicolumn{1}{|c|}{ Exemplos de "Pontos de Escuta" } \\
\hline 1. Universidades \\
\hline 2. CENTECs-Centro Tecnológico do Ceará \\
\hline 3. CVTs-Centro Vocacional Tecnológico \\
\hline 4. SENAI-Serviço Nacional da Indústria \\
\hline 5. EMBRAPA- Empresa Brasileira de Pesquisa Agropecuária \\
\hline 6. CIN-Centro Internacional de Negócios-(Federação das Indústrias do Ceará) \\
\hline 7. Centros Culturais \\
\hline 8. Associações de classe e comunitárias \\
\hline 9. Centros de Estudos e Pesquisas Internacionais \\
\hline 10. Câmaras de Comércio \\
\hline 11. Sindicatos \\
\hline 12. Bancos \\
Fonte: Elaborado pelas autoras
\end{tabular}


Diagrama 2: Tecnologia de mobilização social por meio de uma abordagem cooperativa

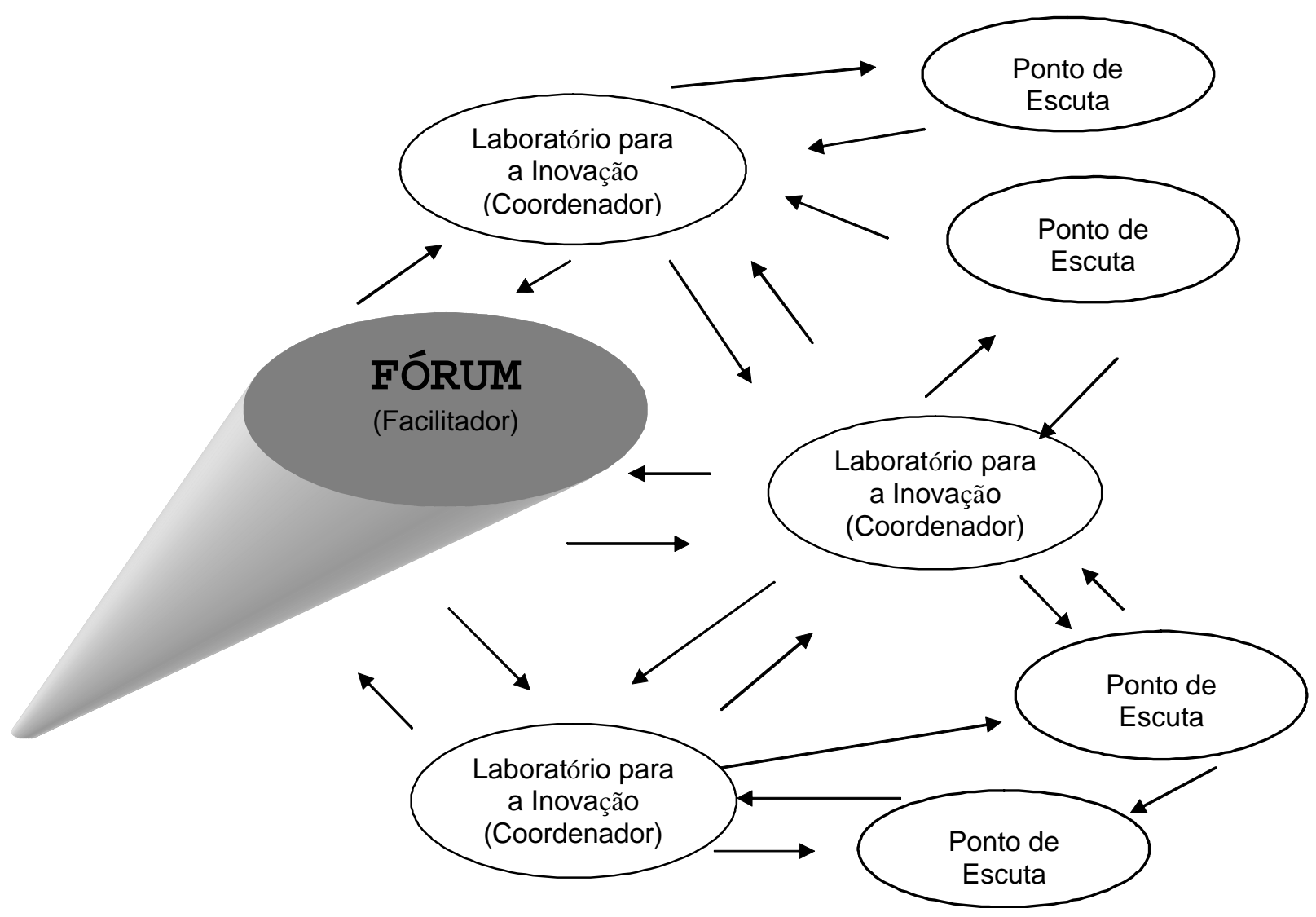

O diagrama acima mostra que as três entidades organizacionais estão intensamente inter-relacionadas constituindo uma rede de relações que fortalecem a interdependência produtiva, exercitando uma colaboração eficiente com a circulação da informação e produção do saber local. A partir desse interrelacionamento e à medida que as conversações entre os técnicos e atores envolvidos avançam são definidos os conjuntos de ações através de uma metodologia que possibilite a aprendizagem cooperativa. Durante todo o processo o gerenciamento das metodologias é realizado através da utilização de instrumentos, com a formulação de indicadores e mapeamentos empíricos, de avaliação e verificação dos impactos, interfaces e implicações no território. Na medida em que facilita o inter-relacionamento, o aprendizado e a inovação, a constituição e a vivência prática dessa rede de inter-relações pode se constituir em um mecanismo eficaz para facilitar a evolução do APL para uma estrutura mais complexa como os SPL.
A idéia é que tudo se inicie com o fórum e a partir dele os grupos de trabalhos sejam formados por temas surgidos nas discussões entre os atores. Esses grupos atuarão como laboratórios de inovação; esses, por sua vez identificarão e se conectarão aos pontos de escuta, fonte privilegiada de informações e experiências relacionados ao tema de cada laboratório. Cada um dessas entidades acima abriga conjuntos de atores produtivos, institucionais e comunitários, assim como em cada uma delas opera uma liderança. Por exemplo, o fórum é liderado por um coordenador que entre outras missões coordena o esforço coletivo de mudança e capitaneia as iniciativas voltadas para esse fim. No caso dos laboratórios, o líder terá o papel de juntar os demais colaboradores do grupo com o intuito de assegurar que as medidas sugeridas pelo fórum sejam levadas adiante. Nos pontos de escuta serão identificados indivíduos de referência para facilitar os contatos e as trocas de informações. Todo o esforço gira em torno de facilitar a mudança e a ino- 
vação - essenciais para a passagem de APL para SPL - e não simplesmente resolver problemas do dia-a-dia.

\section{Considerações finais}

Primeiramente este trabalho procurou fazer uma discussão ampla das diversas formas de abordagem sobre aglomerações de MPEs. Destaca-se dentre estas abordagens aquela denominada Arranjos Produtivos Locais (APLs) e suas possibilidades de evolução para os Sistemas Produtivos Locais (SPLs), foco de análise deste estudo. A partir deste enfoque geral, foi elaborada e proposta uma tecnologia de mobilização dos APLs, enfocando a construção e fortalecimento do capital social e da governança.

A tecnologia de mobilização dos Arranjos Produtivos Locais (APLs) apresentada neste estudo ainda está em construção teórica. Contudo, a intenção prática é aquela de discutir projetos focalizados e ações integradas de suporte regional sobre objetivos de desenvolvimento compartilhados em nível local a partir dos recursos disponíveis na região. Considerando a profundidade da transformação, estes poderão realizar-se somente em um período a médio e longo prazo. O importante será colocar em movimento um processo que desencadeie rapidamente alguns primeiros resultados e que, para o restante das iniciativas, permita de definir os novos objetivos a serem alcançados por prazos definidos. Ressalta-se que uma política por parte dos poderes públicos, de coletividade local, de associações de cidadãos e de grupos de empresários pode melhor conectar os APLs ao desenvolvimento ecossistêmico das regiões explorando ao máximo as potencialidades do capital social e da governança, através da valorização e criação de sinergias entre as competências locais, a organização das complementaridades entre recursos e projetos, as trocas de saberes e de experiências, a formação de redes de ajuda mútua, a auto-organização das comunidades locais, a maior participação dos atores e da população em geral nas decisões políticas, a abertura para novas formas de especialidades e parcerias como meios para viabilizar o desenvolvimento local da região/cidade.

\section{Notas:}

${ }^{1}$ A chamada Terceira Itália constitui a região intermediária entre o Norte e o Sul do país. A partir dos anos 70, essa região passou a alcançar forte dinamismo, gerado principalmente por conta de aglomerações de pequenas empresas, conhecidas como distritos industriais, que se especializaram em negócios específicos.

${ }^{2}$ Arranjos produtivos locais podem ser definidos como sendo "aglomerações territoriais de agentes econômicos, políticos e sociais, com foco em um conjunto específico de atividades econômicas e que apresentam vínculos e interdependência" (ALBAGLI e BRITO, 2002, p. 3).

${ }^{3}$ Sistemas produtivos e inovativos locais são definidos como "arranjos produtivos cuja interdependência, articulação e vínculos consistentes resultam em interação, cooperação e aprendizagem, possibilitando inovações de produtos, processos e formatos organizacionais e gerando mais competitividade empresarial e capacitação social" (ALBAGLI e BRITO, 2002, p. 3).

\section{Referências bibliográficas}

ALBAGLI, S.; BRITO, J. Arranjos Produtivos Locais: uma nova estratégia de ação para o SEBRAE - Glossário de Arranjos Produtivos Locais. Rede Sist, 2002.

AMARAL FILHO, J. do. A endogeinização no desenvolvimento econômico regional. ENCONTRO NACIONAL DA ANPEC, 27., dezembro 1999, BelémPA. Anais... Belém, 1999. p. 1281-1300.

AUDRETSCH, D. B. Agglomeration and the location of innovative activity. Oxford Review of Economic Policy 14: 2,Verão 1998.

BAPTISTA, Creomar. Distritos flexiveis e desenvolvimento endógeno: uma abordagem "marshalliana". Disponível em: <http://www.geocities.com/statprof/distrit.html>. Acesso em: 20 jan. 2003.

BRITTO, Jorge; ALBUQUERQUE, Eduardo da Motta. Características estruturais de Clusters Industriais na economia brasileira: uma análise inter-setorial. Disponível em: <http://www.nepp.unicamp.br/ cadernos/cadernos/caderno39.pdf >. Acesso em: 22 jan. 2003.

CASSIOLATO, J.E.; SZAPIRO, Marina. Proposição de políticas para a promoção de sistemas produtivos locais de micro, pequenas e médias empresas - arranjos e sistemas produtivos locais no Brasil. Rio de Janeiro: Instituto de Economia da Universidade Federal do Rio de Janeiro - IE/UFRJ, 2002.

KRUGMAN, P. What's new about the new economic geography? Oxford Review of Economic Policy 14: 2, Verão 1998.

LE BORGNE, D. La politique industrielle regionale en Italie. Paris: Ministère de l'industrie et de l'Aménagement du Territoire/CEPREMAP, 1991.

MARSHALL, Alfred. Princípios de economia. São Paulo: Nova Cultural, 1996.

PORTER, M. E. Clusters and the new economics of competition. Harvard Business Review, nov./dez. 1998. 
SCHMITZ, Hubert. Collective efficiency: growth path for small-scale industry. Brighton: IDS, 1994.

SCHMITZ, Hubert; NADVI, Khalid. Clustering and Industrialization: Introduction. World Development, v. 27, n. 9, p. 1503-1514, 1999.
SCOTT, A. The geographic foundations of industrial performance. In: CHANDLER Jr., A.; HAGSTROM, P.; SOLVELL, O. (eds.). The dynamic firm - the role of technology, organization and regions. Oxford: Oxford University Press, 1998. Chapter 16. 